\title{
Cepas invasivas de pneumococo isoladas de crianças e adolescentes em Salvador
}

\author{
Invasive pneumococcal strains isolated from children and adolescents in Salvador \\ Cristiana M. Nascimento-Carvalho ${ }^{1}$, Leda S. Freitas-Souza ${ }^{1}$, Otávio A. Moreno-Carvalho ${ }^{2}$, \\ Noraney N. Alves ${ }^{3}$, Renilza M. Caldas ${ }^{3}$, Maria G. Barberino ${ }^{4}$, Jussara Duarte ${ }^{4}$, Maria A. Brandão ${ }^{4}$, \\ Dilton R. Mendonça ${ }^{5}$, Adriana Silva ${ }^{5}$, Maria L. Guerra ${ }^{6}$, Maria C. Brandileone ${ }^{7}$, José L. Di Fabio ${ }^{8}$
}

\section{Resumo}

Objetivos: descrever resistência antimicrobiana e sorotipos de cepas de pneumococo.

Métodos: durante 57 meses, foi conduzida uma vigilância de cepas invasivas de pneumococo de pacientes com idade $<20$ anos. O pneumococo foi identificado pelos testes de solubilidade da bile e optoquina. A resistência à penicilina foi avaliada com o disco de oxacilina $(1 \mu \mathrm{g}) \mathrm{e}$, para as cepas não suscetíveis, foi determinada a concentração inibitória mínima. Provas de difusão de disco e de microdiluição em placa foram utilizadas para avaliação da resistência a outros antimicrobianos. Reação de Neufeld-Quellung foi realizada para sorotipagem.

Resultados: dos 70 pacientes, $57,1 \%$ eram do sexo masculino. A mediana da idade foi 1,92 anos (média 3,19 $\pm 3,66$ anos, variando de um mês a 19,5 anos), tendo $52,9 \%$ e $81,4 \%$ dos pacientes idade $<2$ anos e $<5$ anos, respectivamente. As cepas foram isoladas do sangue $(91,4 \%)$, liquor (2,9\%), líquidos pleural (2,9\%), peritonial $(1,4 \%)$ ou de abscesso $(1,4 \%)$, de pacientes com pneumonia $(77,1 \%)$, febre sem sinais localizatórios $(10,0 \%)$, meningite $(4,3 \%)$ e outros diagnósticos $(8,6 \%)$. Resistência foi detectada à penicilina $(20,0 \%)$, ao sulfametoxazol-trimetoprim $(65,7 \%)$, à tetraciclina $(21,4 \%)$, à ofloxacina $(6,3 \%)$, à eritromicina $(5,7 \%)$ e à clindamicina $(2,9 \%)$. Não houve resistência ao cloranfenicol ou à vancomicina. Entre as cepas não suscetíveis à penicilina, resistência absoluta foi detectada em uma, a mesma que apresentou resistência intermediária à cefotaxima. Os sorotipos mais frequientes foram: 14 (22,9\%), 5 e $6 \mathrm{~A}$ ( $10,0 \%$ cada), $6 \mathrm{~B}$ e $19 \mathrm{~F}$ ( $8,6 \%$ cada), $9 \mathrm{~V}, 18 \mathrm{C}$ e $23 \mathrm{~F}(5,7 \%$ cada). Das cepas resistentes à penicilina, $71,4 \%$ pertenciam ao sorotipo $14,14,3 \%$ aos sorotipos $6 \mathrm{~B}$ e $19 \mathrm{~F}$ (cada).

Conclusões: das 70 cepas, $67,2 \%$ têm sorotipos incluídos na vacina pneumocócica heptavalente conjugada, assim como estão incluídos todos os sorotipos que apresentaram resistência à penicilina.

J Pediatr (Rio J) 2003;79(3):209-14: Streptococcus pneumoniae, resistência antimicrobiana, sorotipos, doença invasiva, criança, adolescente.

\begin{abstract}
Objectives: describe the antimicrobial resistance and serotype distribution of pneumococcal strains.

Methods: in a 57-month period, a laboratory-based surveillance of invasive pneumococcal strains from patients aged $<20$ years was conducted. Pneumococcus was identified by means of tests for solubility in bile and optochin. Pneumococcal resistance to penicillin was screened by $1 \mu \mathrm{g}$ oxacillin disc and minimal inhibitory concentration was determined for the strains not susceptible to penicillin. Disc diffusion and broth microdilution methods were used for surveillance of resistance to other antimicrobials. Pneumococci were serotyped by means of the Neufeld-Quellung reactions.
\end{abstract}

Results: of 70 patients, $57.1 \%$ were males. The mean age was 1.92 yrs (mean $3.19+3.66$ yrs, range 1 month to $19.5 \mathrm{yrs}$ ); $52.9 \%$ and $81.4 \%$ were $<2$ yrs and $<5 \mathrm{yrs}$, respectively. The strains were isolated from blood $(91.4 \%)$, CSF $(2.9 \%)$, pleural $(2.9 \%)$, peritoneal $(1.4 \%)$ and abscess $(1.4 \%)$ fluids from patients with pneumonia $(77.1 \%)$, fever without localizing signs $(10.0 \%)$, meningitis $(4.3 \%)$, others $(8.6 \%)$. Resistance was detected to penicillin (20.0\%), trimethoprimsulfamethoxazole $(65.7 \%)$, tetracycline $(21.4 \%)$, ofloxacin $(6.3 \%)$, erythromycin $(5.7 \%)$, clindamycin $(2.9 \%)$. All tested strains were susceptible to chloramphenicol and vancomycin. Among penicillinresistant strains, high resistance was detected in one, the same that showed intermediate resistance to cefotaxime. The most frequent serotypes were: 14 (22.9\%), 5 and $6 \mathrm{~A}$ (10.0\% each), 6B and $19 \mathrm{~F}(8.6 \%$ each), $9 \mathrm{~V}, 18 \mathrm{C}$ and $23 \mathrm{~F}$ (5.7\% each). Resistance to penicillin was detected in serotypes 14 (71.4\%), 6B and 19F (14.3\% each).

Conclusions: of 70 strains, $67.2 \%$ were classified as serotypes included in the heptavalent conjugate pneumococcal vaccine as well as were all penicillin-resistant strains.

J Pediatr (Rio J) 2003;79(3):209-14: Streptococcus pneumoniae, antimicrobial resistance, serotypes, invasive disease, children, adolescents.

1. Doutora - Universidade Federal da Bahia, BA.

2. Liquorologista - Laboratório de Liquor - SINPEL / Fundação José Silveira, BA.

3. Bacteriologista - Universidade Federal da Bahia, BA.

4. Bacteriologista - Hospital Aliança, BA.

5. Pediatra - Hospital Central Roberto Santos.

6. Biomédica - Instituto Adolfo Lutz, SP.

7. Doutora - Instituto Adolfo Lutz, SP.

8. Doutor - Organização Panamericana de Saúde, Washington DC, EUA.

Este estudo recebeu apoio técnico-material do Ministério da Saúde e da Organização Panamericana de Saúde.

Artigo submetido em 10.12.02, aceito em 26.03.03. 


\section{Introdução}

O Streptococcus pneumoniae é um agente etiológico importante, especialmente de pneumonia, meningite e sepse, com ênfase na faixa etária pediátrica, podendo causar doença grave ${ }^{1}$. A Organização Mundial de Saúde estima que, anualmente, entre 1 e 2 milhões de óbitos ocorrem em crianças menores que cinco anos, em decorrência de infecção pneumocócica, em todo o mundo, sendo que a maioria destes óbitos são registrados em países em desenvolvimento $^{2}$.

A mortalidade por infecção pneumocócica, inclusive por pneumonia pneumocócica, sofreu grande declínio após a introdução das sulfonamidas e da penicilina, entre os anos 30 e 40 do século passado ${ }^{3}$. As primeiras cepas de pneumococo resistentes às sulfonamidas foram relatadas já em $1943^{4}$. Em 1965 foi descrita, pela primeira vez, a ocorrência de cepa de $S$. pneumoniae resistente à penicilina ${ }^{5}$. Desde então, particularmente nos últimos dez anos, tem sido cada vez mais freqüente, em diversas partes do mundo, o relato de casos de infecção causada por pneumococo com suscetibilidade diminuída, ou até mesmo totalmente resistente à penicilina e/ou a outros antimicrobianos utilizados no tratamento desta infecção ${ }^{6-11}$, inclusive no Brasil ${ }^{12-14}$.

Em virtude dos dados anteriormente expostos, o uso de uma vacina pneumocócica conjugada, imunogênica para crianças a partir dos dois meses de vida, tem sido considerada uma estratégia potencial para o controle das infecções pneumocócicas ${ }^{15,16}$. No entanto, mais de 90 sorotipos de pneumococo já foram identificados, e vários são os sorotipos que causam doença ${ }^{17}$. O perfil de sorotipos mais prevalentes difere de uma região para outra ${ }^{18} \mathrm{e}$ a imunidade induzida aparentemente é sorotipo-específica ${ }^{15}$. Portanto, para o amplo uso da vacina pneumocócica conjugada, é necessário o adequado conhecimento da distribuição dos sorotipos mais prevalentes em cada região, assim como é necessário conhecer-se a suscetibilidade do pneumococo aos antimicrobianos para a escolha correta dos esquemas terapêuticos a serem utilizados no tratamento desta infecção ${ }^{19}$.

O objetivo desta investigação foi descrever o padrão de resistência antimicrobiana e a distribuição de sorotipos de cepas invasivas de pneumococo, isoladas de uma amostra de crianças e adolescentes em Salvador, Bahia.

\section{Métodos}

\section{Desenho do estudo e população}

Entre setembro de 1997 e maio de 2002, foi conduzida uma vigilância ativa sobre cepas invasivas de pneumococo. Participaram do estudo os laboratórios de bacteriologia do complexo hospitalar Hospital Universitário Professor Edgard Santos - Centro Pediátrico Professor Hosannah de Oliveira (HUPES-CPPHO), Hospital Aliança (HA), durante todo o período, Hospital Central Roberto Santos (HCRS) durante o ano de 1999, e Laboratório de Liquor do Serviço de Infectologia Pediátrica e Liquorologia da Bahia - Fundação José Silveira (SINPEL-FJS), durante os anos de 2001 e 2002, em Salvador, Bahia. Foram coletados dados clínico-demográficos dos pacientes de cujos fluidos estéreis as cepas de pneumococo foram isoladas, a partir dos dados registrados nos pedidos das culturas ou, quando necessário, a partir de entrevista com o médico assistente, ou consulta ao prontuário médico, imediatamente após o isolamento de cada cepa invasiva de pneumococo. Incluiu-se neste estudo as cepas isoladas de pacientes com idade inferior a 20 anos.

\section{Dados bacteriológicos}

No laboratório do HUPES-CPPHO, até 1999, e no SINPEL, o fluido biológico a ser cultivado foi imediatamente inoculado $(1,0-3,0 \mathrm{ml})$ em $30 \mathrm{ml}$ de infusão de cérebro-coração (BHI) com SPS $(0,025 \%)$ e incubado a $35^{\circ} \mathrm{C}$. Todas as culturas foram submetidas à subcultura em ágar-sangue de carneiro a $5 \%$ e ágar-chocolate, a $35^{\circ} \mathrm{C}$, após $24 \mathrm{~h}, 48 \mathrm{~h}$ e 7 dias de incubação no BHI. No laboratório do HUPES-CPPHO (a partir de 2000), do HA e do HCRS, 0,5-4,0 $\mathrm{ml}$ dos espécimes coletados foram imediatamente inoculados em $20 \mathrm{ml}$ de BHI suplementado e incubados no aparelho Bact/Alert da Organon, a $35^{\circ} \mathrm{C}$, durante 7 dias. Sempre que o aparelho sinalizava um resultado positivo, o meio era submetido à subcultura em ágar-Columbia, com $5 \%$ de sangue de carneiro, e em ágar-chocolate, incubados a $35^{\circ} \mathrm{C}$ e em $5 \% \mathrm{CO}_{2}$, por 18-24 horas. S. pneumoniae foi distinguido de outros estreptococos alfa-hemolíticos por meio dos testes de solubilidade em bile e optoquina. As cepas de pneumococo foram encaminhadas para o Laboratório Central da Bahia (LACEN-BA) e, em seguida, para o Instituto Adolfo Lutz, em São Paulo, onde a identificação bacteriológica foi confirmada, e os testes de suscetibilidade antimicrobiana e a sorotipagem foram realizados. A resistência do pneumococo à penicilina foi identificada inicialmente com o uso do disco de oxacilina $1 \mu \mathrm{g}$. O método de difusão do disco foi utilizado para a investigação de resistência a outros antimicrobianos, incluindo cloranfenicol, sulfametoxazol-trimetoprim, eritromicina, clindamicina, ofloxacina, vancomicina e tetraciclina. As cepas com suscetibilidade diminuída à oxacilina (zona de inibição de crescimento bacteriano em volta do disco de oxacilina inferior a 20mm) foram submetidas à determinação da concentração inibitória mínima (MIC) para penicilina e para cefotaxima, por meio do método de microdiluição em placa (Mueller-Hinton suplementado com 2\%-5\% de hemácias lisadas de cavalo $)^{20}$. Foram utilizados os seguintes valores de MIC para a definição da suscetibilidade à penicilina: $\mathrm{MIC} \leq 0,06 \mu \mathrm{g} / \mathrm{ml}-$ suscetível; $0,12 \mu \mathrm{g} \leq \mathrm{MIC}$ $\leq 1,00 \mu \mathrm{g}$ - resistência intermediária; MIC $\geq 2,00 \mu \mathrm{g} / \mathrm{ml}-$ resistência absoluta ${ }^{20}$. A sorotipagem do pneumococo foi realizada por meio da reação de Neufeld-Quellung utilizando anti-soro produzido pelo Statens Seruminstitut, Copenhagem, Dinamarca. 


\section{Local do estudo}

Os laboratórios de bacteriologia do HUPES-CPPHO, HA e HCRS realizam exames de pacientes atendidos nos respectivos hospitais. O HUPES-CPPHO é um hospital público e universitário, localizado na área central de Salvador, e atende pacientes predominantemente de classe socioeconômica baixa, procedentes de Salvador e cidades vizinhas. O HCRS é público, está localizado em bairro residencial de classes média baixa e baixa e atende principalmente pacientes de classe socioeconômica baixa. O HA está localizado em zona residencial de classes socioeconômicas média e alta e atende pacientes dessas classes. O SINPELFJS realiza exames de liquor de pacientes atendidos em dezessete hospitais da cidade, pertencentes às classes socioeconômicas baixa, média e alta, procedentes de Salvador e de outras cidades do estado da Bahia. De acordo com um estudo sobre pneumonia comunitária em crianças e adolescentes, conduzido no CPPHO e no HA, entre 1997 e 1999, 7,4\% das crianças hospitalizadas com pneumonia em Salvador são internadas nestes dois hospitais ${ }^{21}$, nos quais a taxa de coleta de hemocultura, neste mesmo grupo de pacientes, é de $65,5 \%$, sendo o pneumococo isolado em $0,8 \%$ das amostras 22 .

\section{Análise dos dados}

A análise estatística foi descritiva, utilizando-se o Statistical Package for Social Sciences (SPSS 9.0). Como o objetivo principal foi descrever o padrão de resistência antimicrobiana do pneumococo invasivo com ênfase para penicilina, o cálculo do tamanho amostral foi realizado considerando-se taxa de resistência de $20 \%$, de acordo com De Cunto Brandileone MC et al. ${ }^{13}$, intervalo de confiança $95 \%$, largura de $20 \%$, encontrando-se, como tamanho mínimo da amostra, 62 cepas $^{23}$. Para a realização deste estudo, obteve-se o consentimento da diretoria de cada hospital ou serviço ao qual o respectivo laboratório sentinela está vinculado. Este estudo integra a rede de vigilância epidemiológica do Ministério da Saúde - Organização Panamericana de Saúde, para S. pneumoniae (SIREVAVIGIA) $^{24}$, na Bahia.

\section{Resultados}

Foram isoladas 70 cepas de pneumococo: $55,7 \%$ no HUPES-CPPHO, $41,5 \%$ no HA, $1,4 \%$ no SINPEL e $1,4 \%$ no HCRS. A idade dos 70 pacientes variou de um mês a 19,5 anos, com mediana de 1,92 anos (média 3,19 + 3,66 anos); respectivamente, $52,9 \%$ e $81,4 \%$ dos pacientes apresentaram idade $<2$ anos e $<5$ anos; $57,1 \%$ dos pacientes pertenciam ao sexo masculino. As cepas foram isoladas do sangue $(91,4 \%)$, liquor $(2,9 \%)$, líquido pleural $(2,9 \%)$, líquido peritonial $(1,4 \%)$ e líquido de abscesso $(1,4 \%)$. Os diagnósticos dos pacientes foram pneumonia $(77,1 \%)$, febre sem sinais localizatórios (FSSL) $(10,0 \%)$, meningite $(4,3 \%)$, celulite $(2,9 \%)$, otite média aguda $(2,9 \%)$, sinusite $(1,4 \%)$ e peritonite $(1,4 \%)$. Dos 64 pacientes em que se isolou pneumococo do sangue, um apresentou-se com meningite. Entre as 70 cepas, resistência à penicilina foi detectada em $20,0 \%$, ao sulfametoxazol-trimetoprim em $65,7 \%$, à tetraciclina em $21,4 \%$, à eritromicina em $5,7 \%$, à clindamicina em 2,9\%. Entre as 64 cepas estudadas para suscetibilidade à ofloxacina, quatro $(6,3 \%)$ mostraram-se resistentes, enquanto que, entre as 17 estudadas para suscetibilidade à cefotaxima, uma $(5,9 \%)$ foi considerada resistente. Todas as 70 cepas foram suscetíveis, quando avaliadas em relação ao cloranfenicol, assim como as 44 cepas estudadas em relação à vancomicina. Entre as cepas não suscetíveis à penicilina, resistência absoluta foi detectada em uma, a mesma que também apresentou resistência intermediária à cefotaxima. A mediana da idade dos pacientes cujas cepas foram resistentes à penicilina foi 2,75 anos (média 4,22 $\pm 4,74$ anos), sendo que $35,7 \%$ apresentaram idade inferior a dois anos, e 78,6\%, idade inferior a cinco anos. A Tabela 1 apresenta a distribuição dos sorotipos e respectivas frequiências de resistência à penicilina. A cepa com resistência absoluta foi classificada no sorotipo 14.

\section{Discussão}

Chama atenção, entre os resultados apresentados, a elevada freqüência de cepas de pneumococo isoladas de sangue $(91,4 \%)$, quando comparada com a frequiência de

Tabela 1 - Distribuição de sorotipos de cepas invasivas de pneumococo e resistência à penicilina, Salvador, 1997-2002

\begin{tabular}{|c|c|c|c|}
\hline \multirow[b]{2}{*}{ Sorotipo } & \multicolumn{2}{|c|}{ Freqüência (\%) } & \multirow{2}{*}{$\begin{array}{c}\text { Resistência à } \\
\text { penicilina }(\%) *\end{array}$} \\
\hline & Válida* & Cumulativa & \\
\hline 14 & $22,9(16 / 70)$ & 22,9 & $71,4(10 / 14)$ \\
\hline 5 & $10,0(7 / 70)$ & 32,9 & - \\
\hline $6 \mathrm{~A}$ & $10,0(7 / 70)$ & 42,9 & \\
\hline $6 \mathrm{~B}$ & $8,6(6 / 70)$ & 51,5 & $14,3(2 / 14)$ \\
\hline $19 \mathrm{~F}$ & $8,6(6 / 70)$ & 60,1 & $14,3(2 / 14)$ \\
\hline $9 \mathrm{~V}$ & $5,7(4 / 70)$ & 65,8 & \\
\hline $18 \mathrm{C}$ & $5,7(4 / 70)$ & 71,5 & \\
\hline $23 \mathrm{~F}$ & $5,7(4 / 70)$ & 77,2 & \\
\hline 3 & $4,3(3 / 70)$ & 81,5 & \\
\hline $10 \mathrm{~A}$ & $4,3(3 / 70)$ & 85,8 & \\
\hline $15^{\dagger}$ & $4,3(3 / 70)$ & 90,1 & \\
\hline $19 \mathrm{~A}$ & $4,3(3 / 70)$ & 94,4 & \\
\hline 1 & $1,4(1 / 70)$ & 95,8 & \\
\hline $9 \mathrm{~N}$ & $1,4(1 / 70)$ & 97,2 & \\
\hline $18 \mathrm{~A}$ & $1,4(1 / 70)$ & 98,6 & \\
\hline 34 & $1,4(1 / 70)$ & 100,0 & \\
\hline Total & 100,0 & 100,0 & 100,0 \\
\hline
\end{tabular}


cepas isoladas em outros fluidos corpóreos normalmente estéreis $(8,6 \%)$. Este achado indica a prática de coleta de hemocultura em pacientes com suspeita de doença bacterêmica, nos hospitais que participaram deste estudo, não se podendo afirmar, contudo, a freqüência com que se executou esse procedimento. Sabe-se apenas que, em dois hospitais (CPPHO e HA), entre 1997 e 1999, hemocultura foi coletada em $65,5 \%$ dos pacientes com pneumonia e idade inferior a $15 \operatorname{anos}^{22}$. É importante ressaltar que, entre as diversas situações em que o pneumococo pode causar doença bacterêmica, merecem destaque a pneumonia, a sepse e a bacteremia oculta, situações estas mais freqüentes do que as meningites ${ }^{1}$. Apesar da elevada letalidade e morbidade das meningites pneumocócicas ${ }^{25}$, muitas das quais representando estágios subseqüentes de doença bacterêmica, as doenças bacterêmicas sem meningite são mais prevalentes ${ }^{26}$. Ademais, há relatos de que a distribuição de sorotipos causadores de bacteremia sem meningite pode ser diferente da distribuição de sorotipos causadores de bacteremia e meningite 27,28 , e de que alguns sorotipos apresentam maior frequiência de resistência antimicrobiana do que outros $^{5,29,30}$. Por conseguinte, informação sobre suscetibilidade antimicrobiana e distribuição de sorotipos de cepas isoladas de sangue é de fundamental importância para a escolha de medidas clínicas e epidemiológicas, como o uso de antimicrobianos e vacinas ${ }^{31}$. O número de exames realizados para a obtenção dos isolamentos aqui apresentados não é uma informação conhecida.

$\mathrm{Na}$ casuística apresentada neste trabalho, $87,1 \%$ das cepas foram isoladas do sangue de pacientes com pneumonia ou FSSL. Também chama atenção o predomínio das infecções pneumocócicas invasivas entre crianças com idade inferior a cinco anos, a maioria delas com idade inferior a dois anos (mediana 1,92 anos), o que está de acordo com o que foi anteriormente documentado por outros autores ${ }^{32,33}$. Mais uma vez, ressalta-se a importância de estratégias preventivas para este tipo de infecção neste grupo etário, entre as quais merece destaque o uso da vacina conjugada, a qual é imunogênica a partir dos dois meses de vida ${ }^{16}$.

Tem sido estimado que entre $5 \%$ e $10 \%$ de crianças com febre apresentam FSSL $^{34}$. Embora a maioria dessas crianças cursem com alguma doença infecciosa aguda autolimitada e benigna ${ }^{35}, 5 \%$ cursam com bacteremia oculta ${ }^{35-38}$, sendo o $S$. pneumoniae o agente mais freqüente desta situação $^{39}$. Ademais, um estudo conduzido por Baron e Fink demonstrou que crianças atendidas em consultório privado têm a mesma chance de ter bacteremia oculta que crianças indigentes ${ }^{36}$. Por isso, ressalta-se a importância de se coletar hemocultura em crianças com FSSL e fatores de risco para bacteremia oculta, tais como: idade inferior a três anos, temperatura axilar $\geq 39^{\circ} \mathrm{C}$, leucócitos $\geq 15.000 / \mathrm{mm}^{3}$ ou com granulações tóxicas e vacuolização, trombocitopenia, doença crônica debilitante, história de contato com doença por Neisseria meningitidis ou Haemophilus influenzae e sonolência ou irritabilidade ${ }^{39-40}$. Este procedimen- to, além de poder fornecer informação para a conduta adequada do paciente, permite o estudo dos sorotipos bacterêmicos de pneumococo.

A taxa de resistência à penicilina aqui relatada é semelhante às taxas relatadas por outros estudos conduzidos em grandes cidades brasileiras ${ }^{13,41}$. Em virtude desta taxa de resistência absoluta ser baixa, conforme Friedland e McCracken, a penicilina cristalina permanece como primeira opção terapêutica para as infecções pneumocócicas sem comprometimento do sistema nervoso central que necessitam de tratamento hospitalar, assim como a amoxicilina permanece como primeira escolha para tratamento ambulatorial das infecções pneumocócicas que prescindem de assistência hospitalar ${ }^{42}$.

Aproximadamente 85,0\% das infecções pneumocócicas que ocorrem na faixa etária pediátrica, em todo o mundo, são causadas por sete sorogrupos: $4,6 \mathrm{~A} / \mathrm{B}, 9 \mathrm{~V}, 14$, $18 \mathrm{C}, 19 \mathrm{~F} \mathrm{e} 23 \mathrm{~F}^{43}$, os mesmos que estão incluídos na vacina conjugada heptavalente, já licenciada para uso ${ }^{44}$. Das cepas aqui apresentadas, $67,2 \%$ pertencem a sorotipos que estão incluídos nesta vacina ${ }^{16}$. Entre os demais sorotipos, o mais freqüente nesta casuística foi o sorotipo cinco $(10,0 \%)$. A importante frequiência do sorotipo cinco já foi demonstrada em investigações realizadas na América do Sul, previamente publicadas ${ }^{9-11,13,41}$. No entanto, nestas mesmas publicações, observou-se a importante freqüência do sorotipo um ${ }^{13,41}$, fato não observado na presente investigação, nem na investigação conduzida na Bahia, por Ko e cols., em que foram estudados pacientes com meningite ${ }^{14}$. Apesar do pequeno tamanho desta casuística, a consonância desta distribuição com os resultados relatados por outros investigadores reforça a urgência de se tornar disponível uma vacina que inclua o sorotipo cinco, para ser utilizada na população geral, oferecendo assim uma proteção mais ampla $^{41}$. Todas as cepas que apresentaram resistência à penicilina neste estudo pertencem a sorotipos contidos na vacina heptavalente conjugada, que assim mostra-se capaz de proteger contra infecções potencialmente mais graves. Desde que a grande vantagem desta vacina é ser imunogênica a partir dos dois meses de vida, e a maior freqüência de infecções pneumocócicas ocorre nos indivíduos com idade inferior a dois anos ${ }^{15}$, os dados obtidos neste trabalho sugerem que a vacina atualmente disponível possa trazer benefícios, na região estudada, a indivíduos que apresentem maior risco de infecção pneumocócica, como os asplênicos ${ }^{45}$, ou maior gravidade de doença pneumocócica, como os pacientes com síndrome da imunodeficiência adquirida $^{46}$.

\section{Referências bibliográficas}

1. Teele DW. Pneumococcal Infections. In: Feigin RD, Cherry JD, editores. Textbook of Pediatric Infectious Diseases. $4^{\mathrm{a}}$ ed. Filadélfia: WB Saunders Company; 1998.p.1129-36.

2. Mulholland K. Strategies for the control of pneumococcal diseases. Vaccine 1999;17 Suppl 1:79-84. 
3. Dowell SF, Kupronis BA, Zell ER, Shay DK. Mortality from pneumonia in children in the United States, 1939 through 1996. N Engl J Med 2000;342:1399-407.

4. Tillett WS, Cambier MJ, Harris WH Jr. Sulfonamide-fast pneumococci: A clinical report of two cases of pneumonia together with experimental studies on the effectiveness of penicillin and tyrothricin against sulfonamide-resistant strains. J Clin Invest 1943;22:249-55.

5. Appelbaum PC. Antimicrobial resistance in Streptococcus pneumoniae: An overview. Clin Infect Dis 1992;15:77-83.

6. Hofmann J, Cetron MS, Farley MM, Baughman WS, Facklam RR, Elliott JA, et al. The prevalence of drug-resistant Streptococcus pneumoniae in Atlanta. N Engl J Med 1995;333: 481-6.

7. Pallares R, Linares J, Vadillo M, Cabellos C, Manresa F, Viladrich $\mathrm{PF}$, et al. Resistance to penicillin and cephalosporin and mortality from severe pneumococcal pneumonia in Barcelona, Spain. N Engl J Med 1995;333:474-80.

8. Center for Disease Control and Prevention. Defining the public health impact of drug-resistant Streptococcus pneumoniae: Report of a working group. MMWR 1996;45:1-20.

9. Rossi A, Ruvinsky R, Regueira M, Corso A, Pace J, Gentile A, et al. Distribution of capsular types and penicillin-resistance of strains of Streptococcus pneumoniae causing systemic infections in Argentinean children under 5 years of age. Microb Drug Resist 1997;3:135-40

10. Castaneda E, Leal AL, Castillo O, De La Hoz F, Vela MC, Arango M, et al. Distribution of capsular types and antimicrobial susceptibility of invasive isolates of Streptococcus pneumoniae in Colombian children. Pneumococcal Study Group in Colombia. Microb Drug Resist 1997;3:147-512.

11. Hortal M, Algorta G, Bianchi I, Borthagaray G, Cestau I, Camou $\mathrm{T}$, et al. Capsular type distribution and susceptibility to antibiotics of Streptococcus pneumoniae clinical strains isolated from Uruguayan children with systemic infections. Microb Drug Resist 1997;3:159-63.

12. Levin AS, Teixeira LM, Sessegolo JF, Barone AA. Resistance of Streptococcus pneumoniae to antimicrobials in São Paulo, Brazil: clinical features and serotypes. Rev Inst Med Trop São Paulo 1996;38:187-92.

13. Brandileone MC, Vieira VS, Casagrande ST, Zanella RC, Guerra ML, Bokermann S, et al. Prevalence of serotypes and antimicrobial resistance of Streptococcus pneumoniae strains isolated from Brazilian children with invasive infections. Pneumococcal Study Group in Brazil for the SIREVA Project. Regional System for Vaccines in Latin America. Microb Drug Resist 1997;3:141-6.

14. Ko AI, Reis JN, Coppola SJ, Gouveia EL, Cordeiro SM, Lôbo TS, et al. Clonally related penicillin-nonsusceptible Streptococcus pneumoniae serotype 14 from cases of meningitis in Salvador, Brazil. Clin Infect Dis 2000;30:78-86.

15. Eskola J, Anttila M. Pneumococcal conjugate vaccines. Pediatr Infect Dis J 1999;18:543-51.

16. Shinefield HR, Black S. Efficacy of pneumococcal conjugate vaccines in large scale field trials. Pediatr Infect Dis J 2000; 19:394-7.

17. Austrian R, Gold J. Pneumococcal bacteremia with especial reference to bacteremic pneumococcal pneumonia. Ann Intern Med 1964;60:759-76.

18. Sniadack DH, Schwartz B, Lipman H, Bogaerts J, Butler JC, Dagan R, et al. Potential interventions for the prevention of childhood pneumonia: geographic and temporal differences in serotype and serogroup distribution of sterile site pneumococcal isolates from children - implications for vaccine strategies. Pediat Infect Dis J 1995;14:503-10.
19. Klugman KP, Friedland IR. Antibiotic-resistant pneumococci in pediatric disease. Microb Drug Resist 1995;1:5-8.

20. National Committee for Clinical Laboratory Standards. Methods for dilution antimicrobial susceptibility tests for bacteria that grow aerobically. $7^{\mathrm{a}}$ ed. Approved standard. Document M7-A2. Villanova, PA: NCCLS; 2000.

21. Nascimento-Carvalho CMC. Aspectos clínicos e bacteriológicos das pneumonias adquiridas na comunidade em crianças e adolescentes na cidade de Salvador [tese]. Salvador (BA): Universidade Federal da Bahia; 2001.

22. Nascimento-Carvalho CMC, Gomes DB, Magalhães MP, Oliveira JR, Vilas-Boas AL, Ferracuti R, et al. Blood cultures from Brazilian pediatric outpatients with community acquired pneumonia. J Trop Pediatr 2002;48:58-60.

23. Hulley SB, Cummings SR. Designing clinical research. $1^{\text {a }}$ ed. Baltimore: Williams \& Wilkins; 1988.p.220.

24. Di Fabio JL, Homma A, Quadros C. Pan American Health Organization Epidemiological Surveillance Network for Streptococcus pneumoniae. Microb Drug Resist 1997;3:131-3.

25. Farhat CK. Meningites Bacterianas Purulentas. In: Farhat CK, Carvalho ES, Carvalho LH, Succi RC. Infectologia Pediátrica. $2^{\mathrm{a}}$ ed. São Paulo: Atheneu; 1998.p.89-103.

26. Fedson DS, Musher DM, Eskola J. Pneumococcal vaccine. In: Plotkin AS, Orenstein WA, editores. Vaccines. $3^{\mathrm{a}}$ ed. Filadélfia: WB Saunders Company; 1998.

27. Gray BM, Converse III GM, Dillon Jr. HC. Serotypes of Streptococcus pneumoniae causing disease. J Infect Dis 1979; 140:979-83.

28. Eskola J, Takala AK, Kela E, Pekkanen E, Kalliokoski R, Leinonen M. Epidemiology of invasive pneumococcal infections in children in Finland. JAMA 1992;268:3323-7.

29. Dowson CG, Coffey TJ, Spratt BG. Origin and molecular epidemiology of penicillin-binding-protein-mediated resistance to B-lactam antibiotics. Trends Microbiol 1994;2:361-5.

30. Klugman KP. Pneumococcal resistance to antibiotics. Clin Microbiol Rev 1990;3:171-96.

31. Musher DM. Infections caused by Streptococcus pneumoniae: clinical spectrum, pathogenesis, immunity and treatment. Clin Infect Dis 1992;14:801-7.

32. Burman LA, Norrby R, Trollfors B. Invasive pneumococcal infections: incidence, presdisposing factors, and prognosis. Rev Infect Dis 1985;7:133-42.

33. Breiman RF, Spika JS, Navarro VJ, Darden PM, Darby CP. Pneumococcal bacteremia in Charleston County, South Carolina: A decade later. Arch Intern Med 1990;150:1401-5.

34. Lorin MI. The febrile child: clinical management of fever and other types of pyrexia. New York: John Wiley \& Sons; 1982.

35. Wright PF, Thompson J, McKee KT Jr, Vaughn WK, Sell SH, Karzon DT. Patterns of illness in the highly febrile young child: epidemiologic, clinical and laboratory correlates. Pediatrics 1981;67:694-700.

36. Baron MA, Fink HD. Bacteremia in private pediatric practice. Pediatrics 1980;66:171-5.

37. Murray DL, Zonana J, Seidel JS, Yoshimori RN, Imagawa DT, St Geme JW Jr. Relative importance of bacteremia and viremia in the course of acute fevers of unknown origin in outpatient children. Pediatrics 1981;68:157-60.

38. Teele DW, Pelton SI, Grant MJ, Herskowitz J, Rosen DJ, Allen $\mathrm{CE}$, et al. Bacteremia in febrile children under 2 years of age: Results of cultures of blood of 600 consecutive febrile children in "walk-in" clinic. J Pediatr 1975;87:227-30.

39. Lorin MI, Feigin RD. Fever without localizing signs and fever of unknown origin. In: Feigin RD, Cherry JD. Textbook of Pediatric Infectious Diseases. $4^{\mathrm{a}}$ ed. Filadélfia: WB Saunders Company; 1998.p.820-3. 
40. Powell KR. Fever without a focus. In: Behrman RE, Kliegman RM, Jenson HB. Nelson Textbook of Pediatrics. $16^{\mathrm{a}}$ ed. Filadélfia: WB Saunders Company; 2000.p.742-3.

41. Di Fabio JL, Castañeda E, Agudelo CI, De La Hoz F, Hortal M, Camou T, et al. Evolution of Streptococcus pneumoniae serotypes and penicillin susceptibility in Latin America, Sireva-Vigía Group, 1993 to 1999. Pediatr Infect Dis J 2001;20:959-67.

42. Friedland IR, McCracken GH, Jr. Management of infections caused by antibiotic-resistant Streptococcus pneumoniae. N Engl J Med 1994;331:377-82.

43. Austrian R. Pneumococcal otitis media and pneumococcal vaccines, a historical perspective. Vaccine 2000;19 Suppl 1:71-7.

44. Black S, Shinefield H, Fireman B, Lewis E, Ray P, Hansen JR, et al. Efficacy, safety and immunogenicity of heptavalent pneumococcal conjugate vaccine in children. Pediatr Infect Dis J 2000;19:187-95.

45. Wong WY, Powars DR, Chan L, Hiti A, Johnson C, Overturf G. Polysaccharide encapsulated bacterial infection in sickle cell anemia: a thirty-year epidemiologic experience. Am J Hematol 1992;39:176-82.
46. Yogev R, Chadwick E. Acquired Immunodeficiency Syndrome (Human Immunodeficiency Virus). In: Behrman RE, Kliegman RM, Jenson HB. Nelson Textbook of Pediatrics. $16^{\mathrm{a}}$ ed. Filadélfia: WB Saunders Company; 2000.p.1026.

\section{Endereço para correspondência}

Dra. Cristiana Nascimento-Carvalho

Departamento de Pediatria, Faculdade de Medicina, Universidade Federal da Bahia

Rua Prof. Aristides Novis, 105/1201B

CEP 40210-630 - Salvador, BA

Fax: (71) 2357.869

E-mail: nascimentocarvalho@hotmail.com 